\title{
Non-monotonic variation of the Kramers point band gap with increasing magnetic doping in BiTel
}

A. M. Shikin ( $\square$ ashikin@inbox.ru )

Saint Petersburg State University

A. A. Rybkina

Saint Petersburg State University

D. A. Estyunin

Saint Petersburg State University

I. I. Klimovskikh

Saint Petersburg State University
A. G. Rybkin

Saint Petersburg State University

S. O. Filnov

Saint Petersburg State University

\section{A. V. Koroleva}

Saint Petersburg State University

\section{E. V. Shevchenko}

Saint Petersburg State University

\section{Yu. Voroshnin}

Saint Petersburg State University

\section{A. E. Petukhov}

Saint Petersburg State University

\section{K. A. Kokh}

Kemerovo State University

O. E. Tereshchenko

Novosibirsk State University

\section{Petaccia}

Elettra Sincrotrone Trieste

\section{G. Di Santo}

Elettra Sincrotrone Trieste

\section{S. Kumar}

Hiroshima Synchrotron Radiation Center

\section{A. Kimura}

Hiroshima University

\section{P. N. Skirdkov}


Prokhorov General Physics Institute of the Russian Academy of Sciences

\section{K. A. Zvezdin}

Prokhorov General Physics Institute of the Russian Academy of Sciences

\section{A. K. Zvezdin}

Prokhorov General Physics Institute of the Russian Academy of Sciences

\section{Research Article}

Keywords: magnetic, rashba, doping, level, bitei, quantum, internal, ordering

Posted Date: August 12th, 2021

DOl: https://doi.org/10.21203/rs.3.rs-798055/v1

License: (c) (i) This work is licensed under a Creative Commons Attribution 4.0 International License. Read Full License

Version of Record: A version of this preprint was published at Scientific Reports on December 1st, 2021. See the published version at https://doi.org/10.1038/s41598-021-02493-8. 


\title{
Non-monotonic variation of the Kramers point band gap with increasing magnetic doping in BiTel
}

\author{
A. M. Shikin 1," , A. A. Rybkina ${ }^{1}$, D. A. Estyunin ${ }^{1}$, I. I. Klimovskikh ${ }^{1}$, A. G. Rybkin ${ }^{1}$, S. O. \\ Filnov $^{1}$, A. V. Koroleva ${ }^{1}$, E. V. Shevchenko ${ }^{1}$, V. Yu. Voroshnin ${ }^{1,2}$, A. E. Petukhov ${ }^{1}$, K. A. \\ Kokh $^{1,3,4}$, O. E. Tereshchenko, $1,5,6$, L. Petaccia ${ }^{7}$, G. Di Santo ${ }^{7}$, S. Kumar ${ }^{8}$, A. Kimura ${ }^{9}$, P. N. \\ Skirdkov ${ }^{10,11}$, K. A. Zvezdin ${ }^{10,11}$, and A. K. Zvezdin ${ }^{10,11}$
}

\author{
${ }^{1}$ Saint Petersburg State University, Saint Petersburg, 198504, Russia \\ ${ }^{2}$ Helmholtz-Zentrum Berlin für Materialien und Energie, BESSY II, D-12489 Berlin, Germany \\ ${ }^{3}$ Kemerovo State University, 650000, Kemerovo, Russia \\ ${ }^{4}$ Sobolev Institute of geology and mineralogy SB RAS, 630090, Novosibirsk, Russia \\ ${ }^{5}$ Novosibirsk State University, Novosibirsk, 630090, Russia \\ ${ }^{6}$ A. V. Rzhanov Institute of Semiconductor Physics, Novosibirsk, 630090, Russia \\ ${ }^{7}$ Elettra Sincrotrone Trieste, Trieste, 34149, Italy \\ ${ }^{8}$ Hiroshima Synchrotron Radiation Center, Hiroshima University, Higashi-Hiroshima, Hiroshima 739-0046, Japan \\ ${ }^{9}$ Graduate School of Science, Hiroshima University, Higashi-Hiroshima, 739-8526, Japan \\ ${ }^{10}$ Prokhorov General Physics Institute of the Russian Academy of Sciences, Moscow, 119991, Russia \\ ${ }^{11}$ Moscow Institute of Physics and Technology, Dolgoprudny, 141700, Russia \\ *ashikin@inbox.ru
}

\section{ABSTRACT}

Polar Rashba-type semiconductor BiTel doped with magnetic elements constitutes one of the most promising platforms for the future development of spintronics and quantum computing thanks to the combination of strong spin-orbit coupling and internal ferromagnetic ordering. The latter originates from magnetic impurities and is able to open an energy gap at the Kramers point (KP gap) of the Rashba bands. In the current work using angle-resolved photoemission spectroscopy (ARPES) we show that the KP gap depends non-monotonically on the doping level in case of V-doped BiTel. We observe that the gap increases with $\mathrm{V}$ concentration until it reaches $3 \%$ and then starts to mitigate. Moreover, we find that the saturation magnetisation of samples under applied magnetic field studied by superconducting quantum interference device (SQUID) magnetometer has a similar behaviour with the doping level. Theoretical analysis shows that the non-monotonic behavior can be explained by the increase of antiferromagnetic coupled atoms of magnetic impurity above a certain doping level. This leads to the reduction of the total magnetic moment in the domains and thus to the mitigation of the KP gap as observed in the experiment. These findings provide further insight in the creation of internal magnetic ordering and consequent KP gap opening in magnetically-doped Rashba-type semiconductors.

\section{Introduction}

Materials and systems that combine the properties of two-dimensional (2D) electron gas with strong spin-orbit coupling and internal ferromagnetism constitute one of the most promising platform for the future development of spintronics and quantum computing $^{1,2}$. One of such material is the polar semiconductor BiTeI doped with magnetic metal atoms. Pure BiTeI has a layered crystal structure formed by the tripple-layer block (TLs) Te-Bi-I separated by van der Waals spacings ${ }^{3-6}$. Electronic structure of this material in the vicinity of the Fermi level is characterized by two parabolic-like energy bands, shifted along $k_{\|}$ from the $\Gamma$-point due to the Rashba effect $\left(E_{ \pm}(k) \sim \frac{\hbar^{2}}{2 m^{*}} k^{2} \pm \alpha_{R} k\right)$. The Rashba parameter $\left(\alpha_{R}\right)$, which is proportional to the $k_{\|}$ shift, is one of the largest $(3.8 \mathrm{eV} \cdot \AA)$ in BiTeI. As a result the parabolic bands are significantly spin split ${ }^{3-8}$. However, spin degeneracy remains only at the parabolas crossing point i.e. Kramers point (KP), since the time reversal symmetry is preserved. Doping of BiTeI with magnetic atoms leads to the lifting of the spin degeneracy and opening of the band gap at the KP (KP gap). The magnetization has to be directed perpendicular to the sample surface to open the gap in all $k_{\|}$. The spin structure of the state becomes "hedgehog"-shape in the vicinity of the KP and the electronic structure can be tuned to have one circular Fermi contour ${ }^{9}$. Similar spin degeneracy lifting can be observed in magnetic topological insulators for the topological surface state $^{10-15}$ or Rashba-like state ${ }^{16}$. 
Systems such as BiTeI with magnetic doping can be used as a basis for highly efficient generation and control of spin currents $^{2,17-22}$. Therefore it can be used as spin filters, high-speed spin switches of memory elements, spin-Hall current devices etc. Moreover, the states with opposite momenta and thus spin orientation can be coupled through the proximity effect with a superconductor. This can lead to the formation of a topologically nontrivial superconducting phase ${ }^{1}$ and consequently, to the emergence of Majorana fermion modes with zero energy ${ }^{23,24}$. The above-mentioned effects in magnetically-doped BiTeI may significantly advance the technology of quantum computing and their solid-state implementation ${ }^{1,25-27}$.

In earlier works, devoted to experimental and theoretical study of $\mathrm{V}$-doped $\mathrm{BiTeI}^{9,28}$, it was shown that the KP gap reaches the value of $\sim 90 \mathrm{meV}$ with the $\mathrm{V}$ concentration of $2 \%$. Remarkably, the ab-initio calculations reveal KP gap no greater than 34 $\mathrm{meV}$ in this case ${ }^{9}$. This discrepancy could be related to unaccounted Bi-vacancies in the vicinity of V impurities, which can affect the magnetic moments of the nearest Te atoms and increase the gap value. KP gap up to $100 \mathrm{meV}$ was also observed in ferroelectric semiconductor GeTe doped with $\mathrm{Mn}$ atoms up to 15\% concentration which becomes a Rahsba-Zeeman system below the Curie temperature ${ }^{29,30}$. However, many features of the KP gap opening in Rashba systems are still weakly studied.

In the present work we study $\mathrm{V}$ and $\mathrm{Mn}$-doped BiTeI samples with impurity concentrations of $0.5 \%, 2 \%, 3 \%, 6 \%$ for $\mathrm{V}$ and $2.5 \%$ for Mn by ARPES, scanning tunneling microscopy (STM), atomic force microscopy (AFM) and SQUID magnetometry. STM studies confirm the homogeneity and the concentration of magnetic impurities over the sample surface. From the analysis of the electronic structure we experimentally show that the KP gap value behaves non-monotonically with increase of doping level. Indeed, initially the gap increases up to $130-135 \mathrm{meV}$ at $3 \%$ of $\mathrm{V}$ impurity. Then the gap saturates and reduces with further increase of the $\mathrm{V}$ concentration to $6 \%$. The theoretical estimations show that such non-monotonic variation of the KP gap size is related to the appearance of antiferromagnetically coupled magnetic impurity $(\mathrm{V}, \mathrm{Mn})$ atoms above $3 \%$ of doping evel in BiTeI. Consequently, the effective magnetic moment at each domain can be reduced, which is accompanied by the decrease in the KP gap value.

\section{Experimental results and discussion}

Fig. 1a shows the ARPES dispersion maps measured for V-doped BiTeI at different V concentrations: $0.5 \% \mathrm{~V}, 2 \% \mathrm{~V}, 3 \% \mathrm{~V}$ and $6 \%$ V. They are accompanied with the second derivatives $\left(d^{2} N / d E^{2}\right)$ representation in Fig. $1 \mathrm{~b}$ for better visualization. For a more precise and quantitative information about the KP gap size variation we analyze the energy distribution curves (EDCs) at the $\Gamma$-point $\left(k_{\|}=0 \AA^{-1}\right)$ which are presented in (c). Here, the decomposition into spectral components is also reported. Blue and purple solid peaks locate the energy positions of the states at the edges of the KP gap. One can see that the KP gap value increases from $\sim 100 \mathrm{meV}$ up to $130-135 \mathrm{meV}$ with the $\mathrm{V}$ concentration from $0.5 \%$ to $3 \%$, respectively. However, further increase in the $\mathrm{V}$-concentration to $6 \%$ leads to a reduction of the KP gap down to $105 \mathrm{meV}$. The overall variation of the KP gap $(2 \Delta)$ for all measured spectra vs V concentration is presented in Fig. 1d. Experimental error of the KP gap estimation is less than 15-25 meV (depending on the ARPES setup; see Methods section) and it is mainly caused by angular broadening (see Ref. ${ }^{12}$ ). Measurements of several samples decree total error and more accurately demonstrate the observed unusual KP gap behaviour. Moreover measured samples were grown separately and tested on different ARPES stations giving similar results of the KP gaps. This reduces the possibility of accidental disturbances in the samples stoichiometry.

We use also a different approach based on the experimentally derived shape of the Rashba bands to distinguish the KP gap value. Initially, we carefully track the position of the bands for all measured spectra using the second derivative along energy (for horizontal features) and momentum (for vertical features) directions. We also improve our estimations by analysing 2D curvature plots of $\mathrm{N}(\mathrm{E})^{31}$. In order to derive the parameters of the Rashba bands we approximate experimental data with the massive Rashba equation:

$$
E_{ \pm}(k)=E_{0}+\frac{\hbar^{2}}{2 m} k^{2} \pm \sqrt{\left(\frac{1}{2} \Delta\right)^{2}+\alpha_{R}^{2} k^{2}}
$$

where $E_{0}$ is the band bottom, $m$ is the effective mass, $\alpha_{R}$ is the Rashba parameter, and $\Delta$ is the momentum-independent KP gap. These approximations are shown by blue curves in Fig. 1a. The resulting KP gap values are $\Delta=70 \mathrm{meV}$ for $0.5 \% \mathrm{~V}$, $\Delta=110 \mathrm{meV}$ for $2 \% \mathrm{~V}, \Delta=120 \mathrm{meV}$ for $3 \% \mathrm{~V}$, and $\Delta=70 \mathrm{meV}$ for $6 \% \mathrm{~V}$. They well fit the observed non-monotonic gap dependence (Fig. 1e) being, though, slightly lower than the KP gaps derived from the EDC analysis (Fig. 1d).

At a closer look, the Rashba equation does not fit the whole doped BiTeI band perfectly (see blue curves in Fig. 1a). Inconsistencies appear at the band's bottom (see Fig. 1a). Indeed, it was realized that the band dispersion of IV-VI semiconductors can be better reproduced with a massive Dirac fermion model. Taken from Ref. ${ }^{29}$ the energy dispersion has the following shape:

$$
E_{ \pm}(k)=E_{0}-\sqrt{m_{D}^{2} v^{4}+\hbar^{2} v^{2} k^{2} \pm \frac{1}{4} \sqrt{\Delta_{D}^{4}+16 \alpha_{D}^{4} k^{2}}}
$$



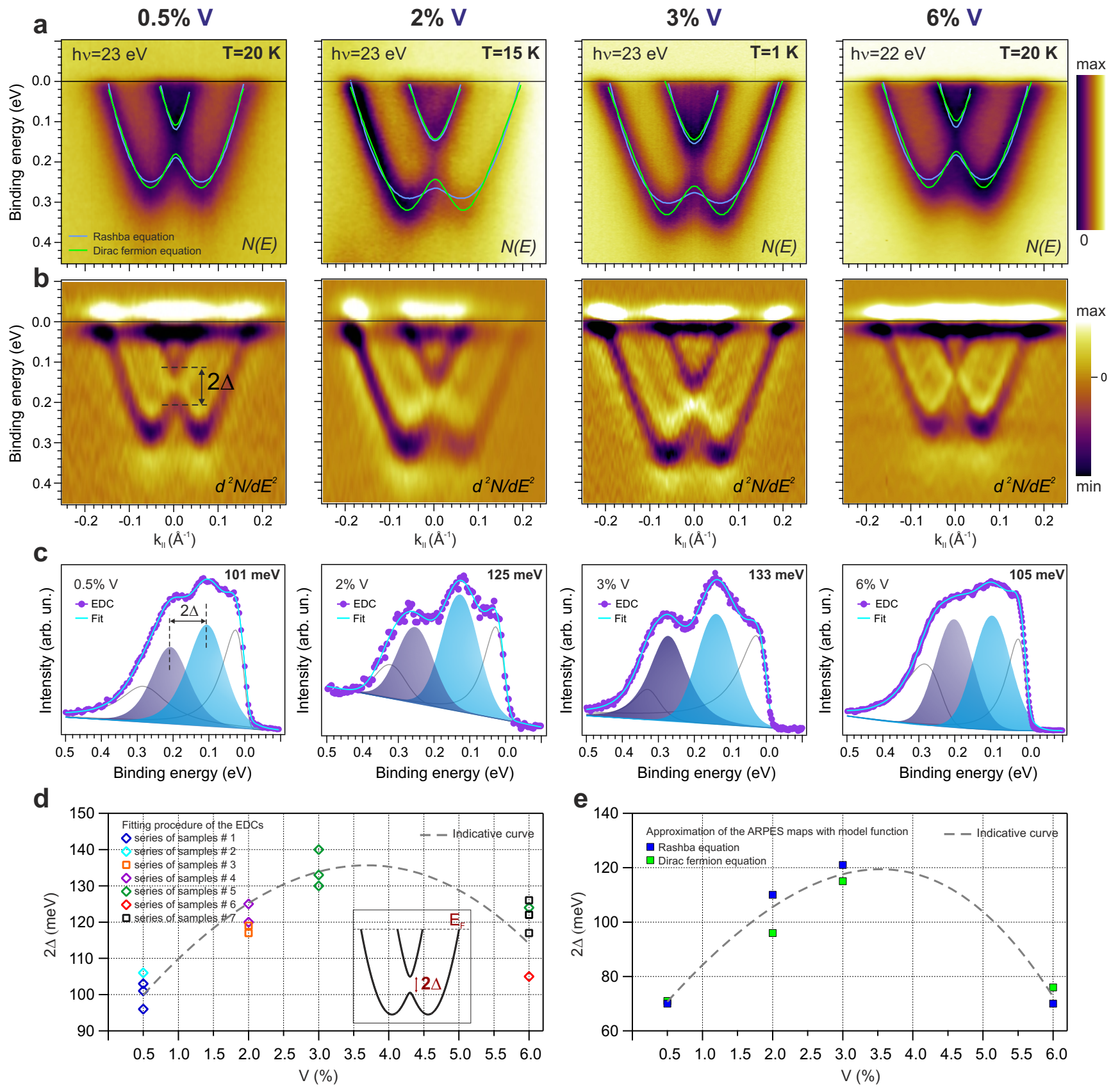

Figure 1. (a) - ARPES dispersion relations $\mathrm{N}(\mathrm{E})$ for V-doped BiTeI with different concentration of V: $0.5 \%$ (T=20 K), $2 \%$ $(\mathrm{T}=15 \mathrm{~K}), 3 \%(\mathrm{~T}=1 \mathrm{~K})$ and $6 \%(\mathrm{~T}=20 \mathrm{~K})$ and their the second derivative $d^{2} N / d E^{2}$ in (b). Approximations of the experimental Rashba bands with two model functions: Rashba equation (eq. 1, blue curves) and Dirac fermion equation (eq. 2, green curves) in (a). (c) - The EDCs cut at the $\Gamma$ point $\left(k_{\|}=0 \AA^{-1}\right)$ with the decomposition into spectral components. The components corresponding to the Rashba parabolas states at the KP gap is shown in blue and purple color. Dependence of the KP gap value $(2 \Delta)$ on $\mathrm{V}$ concentration $(0.5,2,3$ and $6 \%$ ) measured for the series of samples (see details in Method section) derived from the EDC analysis (d) and model functions approximation (e). The dash lines are guide for the eye in (d,e) and the schematic presentation of the measured parameters is shown in inset of (d) 
Here $m_{D}$ is the Dirac mass and represents the curvature for small $k$-values, $v$ is the band velocity and represents the steepness of the bands for larger $k$-values, and $E_{0}$ is a band offset. $\alpha_{D}$ is the Rashba-like parameter and the $\Delta_{D}$ energy term is proportional to $\Delta$ from the massive Rashba equation (i.e. $\Delta=\sqrt{m_{D}^{2} v^{4}+\frac{1}{4} \Delta_{D}^{2}}-\sqrt{m_{D}^{2} v^{4}-\frac{1}{4} \Delta_{D}^{2}}$ ). The massive Dirac fermion model has indeed better consistency with the experimental bands (see green curves in Fig. 1a). Obtained KP gaps within this model are following $\Delta=71 \mathrm{meV}$ for $0.5 \% \mathrm{~V}, \Delta=96 \mathrm{meV}$ for $2 \% \mathrm{~V}, \Delta=115 \mathrm{meV}$ for $3 \% \mathrm{~V}$, and $\Delta=76 \mathrm{meV}$ for $6 \% \mathrm{~V}$. Anyway, both models deliver close results for KP gaps.

One can also notice that the position of the Rashba bands, taken as the binding energy of the band minimum ( $\mathrm{E}_{\text {min }}$ ), is not constant for all measured spectra in Figs. 1a,b. While V-concentration increases till 3\%, the Rashba band positions shift to higher binding energies (i.e. $\mathrm{E}_{\min }$ increases) and reach their maximum shift of about 60 meV. Further increase of $\mathrm{V}$ concentration leads to the backward move of the bands. One can find correlation of such behavior with the one observed for the KP gap (Fig. 1d,e). However, the KP gap size depends on V concentration, while the Rashba bands shift can be also related to differences in temperature or experimental environment.

We have also studied doped BiTeI samples with $3 \% \mathrm{~V}$ and $6 \% \mathrm{~V}$ as well as pure BiTeI using micro $(\mu)$-spot laser based ARPES (hv=6.3 eV). Corresponding dispersion relations accompanied with their EDC curvature plots ${ }^{31}$ are presented in Fig. 2. Laser-ARPES is known to have better spatial, momentum and energy resolution than regular synchrotron based ARPES ${ }^{32}$ (see Methods section). Moreover, misalignment from the normal emission (i.e. the $\Gamma$-point) is less significant at lower energy, which benefits for the precise KP gap estimation. Nevertheless, mean free path of ejected electrons rapidly rises at low photon energy and so additional bulk bands appear in the spectra such as Rashba bands from the next TLs. In Figs. 2a,d,g one can find the second order Rashba band (marked with green squares $-\mathrm{R}_{2}$ ) additionally to the main one (marked with blue circles - $\mathrm{R}_{1}$ ). $\mathrm{R}_{2}$ is visually shifted to the lower binding energy for about $105 \mathrm{meV}(3 \% \mathrm{~V})$ and $130 \mathrm{meV}(6 \% \mathrm{~V})$ due to weaker band bending effect in the 2nd TL. Rashba bands from the next TLs are unresolved, but contribute to the high intensity region near the Fermi level. This region overlaps with the KP of the $\mathrm{R}_{1}$ and $\mathrm{R}_{2}$ bands obstructing EDC analysis of the KP gap. Therefore, here we relay only on the matching of bands with model functions (i.e. gapped Rashba and massive Dirac fermion equations, eq. 1 and 2 respectively). These approximations are presented in Figs. 2c,f,i for both Rashba bands. The parabolic functions of the Rashba model (white curves) fit worse again to the experimental bands than the Dirac model. Though, the fitting discrepancy between the models is significantly less for $\mathrm{R}_{2}$ than for $\mathrm{R}_{1}$, and in case of $3 \% \mathrm{~V}$ spectrum, both approximation results almost coincide. We estimate the KP gaps from the fitting for both $\mathrm{R}_{1}$ and $\mathrm{R}_{2}$ bands for pristine and doped BiTeI. In case of BiTeI the KP gaps for both $\mathrm{R}_{1}$ and $\mathrm{R}_{2}$ bands and both Rashba and Dirac models differ no more than $10 \mathrm{meV}$, which can be taken as value for the $\mathrm{KP}$ gap experimental resolution. For $3 \% \mathrm{~V}$ doped BiTeI the Rashba model provides KP gaps of $\Delta_{1}^{R}=78 \mathrm{meV}, \Delta_{2}^{R}=25 \mathrm{meV}$ while the Dirac model $\Delta_{1}^{D}=62 \mathrm{meV}, \Delta_{2}^{D}=28 \mathrm{meV}$. The KP gaps for $6 \% \mathrm{~V}$ doped BiTeI are $\Delta_{1}^{R}=68 \mathrm{meV}, \Delta_{2}^{R}=20 \mathrm{meV}$ and $\Delta_{1}^{D}=66 \mathrm{meV}, \Delta_{2}^{D}=24 \mathrm{meV}$. One can see that both models reveal close results. However, KP gaps for $3 \% \mathrm{~V}(62 \mathrm{and} 78 \mathrm{meV})$ and for $6 \% \mathrm{~V}(66$ and $68 \mathrm{meV})$ do not differ significantly and demonstrate rather saturation of the KP gap value than decrease. Still it can correlate with synchrotron-derived results, which on average also show saturation-like behaviour between 3 and $6 \% \mathrm{~V}$ in Fig. 1d for some spectra.

The magnetic properties of the V-doped BiTeI and their possible modulation at different V-concentrations have been also investigated using SQUID magnetometry. Fig. 3a-d shows the $M(H)$ magnetization curves for 0.5, 1.75, 3 and $6 \%$ of $\mathrm{V}$ concentrations. The external magnetic field was applied perpendicular to the surface plane i.e. along the $c$ axis. The presented $M(H)$ curves were measured for $0.5 \% \mathrm{~V}$ and $1.75 \% \mathrm{~V}$ at a temperature of $4 \mathrm{~K}$ (Fig. $3 \mathrm{~b}$ ) and for $3 \% \mathrm{~V}$ and $6 \% \mathrm{~V}$ at a temperatures from $2 \mathrm{~K}$ to $30 \mathrm{~K}$ (Fig. 3a,c,d). The magnetization curves in Fig. 3a-d show the $S$-shape behavior up to $30 \mathrm{~K}$ and the paramagnetic linear behavior at $30 \mathrm{~K}$ and above. For all presented V-concentrations the magnetization curves do not show visible hysteresis loop characteristic for ferromagnetic coupling at any measured temperatures but rather the typical behavior for antiferromagnets or paramagnets. Therefore, despite the observed sizable KP gaps by ARPES these materials do not reveal ferromagnetic properties even at $2 \mathrm{~K}$. Nevertheless, the analysis of the saturation moment in Fig. 3a-d shows the increasing of $M$ value in the range of $0.085 \rightarrow 0.13 \rightarrow 0.156 \mathrm{emu} / \mathrm{g}$ with rising of doping level in the range of $0.5 \% \mathrm{~V} \rightarrow 1.75 \% \mathrm{~V} \rightarrow$ $3 \% \mathrm{~V}$. Instead, as the $\mathrm{V}$ concentration goes from $3 \%$ to $6 \%$, the saturation magnetic moment decreases slightly from 0.155 to $0.143 \mathrm{emu} / \mathrm{g}$. This behavior correlates with the non-monotonic dependence of the KP gap size vs V doping reported in Fig. 1 . The temperature dependence of the magnetic susceptibility $(\chi)$ and inverse magnetic susceptibility $\left(\chi^{-1}\right)$ for $3 \% \mathrm{~V}$ and $6 \% \mathrm{~V}$ measured at applied magnetic field of $1 \mathrm{kOe}$ are presented in Fig. 3e,f. The diamagnetic background was subtracted by a linear fit at the high-temperatures data points. Zero-field cooled (ZFC) and field cooled (FC) magnetic susceptibility curves coincide at all temperatures. The inverse magnetic susceptibility curves for both V-concentrations appear to be non-linear from $2 \mathrm{~K}$ to $300 \mathrm{~K}$ which leads to uncertain results of critical temperature by fitting with Curie-Weiss function.

In order to rationalize the results of ARPES measurements, which reveal the KP gap opening requiring ferromagnetic order, and the SQUID measurements, which contradict the latter, we propose the following scenario. Magnetic domains with opposite orientations and size of tens of nanometers are formed in magnetically-doped BiTeI. Since the SQUID signal is integrated over the entire sample, the oppositely oriented magnetic domains may compensate each other on average and lead to a near zero total 

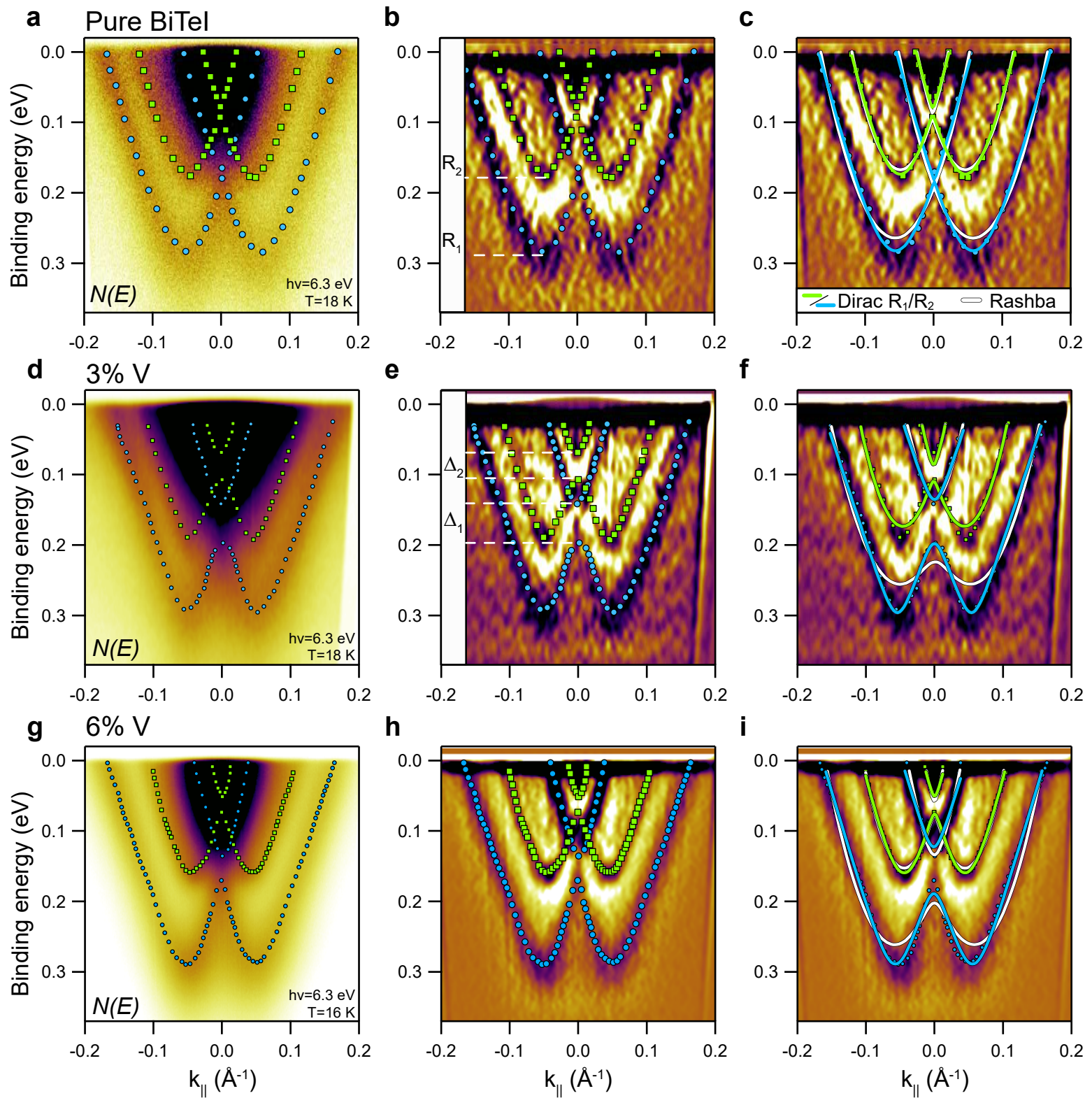

Figure 2. (a), (d), (g) - ARPES dispersion relations $N(E)$ measured with laser photoexitation (hv $=6.3 \mathrm{eV}$ ) for pure BiTeI and for 3\% $\mathrm{V}$ and $6 \% \mathrm{~V}$ doped BiTeI. (b), (e), (h) EDC curvature plots of corresponding $N(E)$ spectra with defined position of the first and second order Rashba states ( $R_{1}$ - blue circles and $R_{2}$ - green squares). $\Delta_{1}$ and $\Delta_{2}$ are KP gaps in the $R_{1}$ and $R_{2}$ states. (c), (f), (i) - Approximation of the experimental points with two model functions: Rashba equation (white curves for both $\mathrm{R}_{1}$ and $R_{2}$ ) and Dirac fermion equation (blue and green for $R_{1}$ and $R_{2}$ respectively). The fitting derived KP gaps for $3 \% \mathrm{~V}$ (f) with the Rashba model are $\Delta_{1}^{R}=78 \mathrm{meV}, \Delta_{2}^{R}=25 \mathrm{meV}$ and with the Dirac model are $\Delta_{1}^{D}=62 \mathrm{meV}, \Delta_{2}^{D}=28 \mathrm{meV}$. The KP gaps for $6 \% \mathrm{~V}$ (i) are $\Delta_{1}^{R}=68 \mathrm{meV}, \Delta_{2}^{R}=20 \mathrm{meV}$ and $\Delta_{1}^{D}=66 \mathrm{meV}, \Delta_{2}^{D}=24 \mathrm{meV}$. 
magnetic moment. As a result, a superparamagnet-like behavior is expected (see, for example, Refs. ${ }^{33-35}$ ), when individual domains have own magnetization and the overall $M(H)$ dependence demonstrates the absence of a clearly visible hysteresis loop. On the other hand, the KP gap opening is indifferent to it, since the gap size is determined by the modulo of the magnetic field of each domain independently on its $\operatorname{sign}^{28}$. Therefore, non-zero KP gaps can be still observed in ARPES as shown in Fig. $1,2$.

Noteworthy, a similar behavior of the KP gap as in Fig. 1d,e was previously observed in Ref. ${ }^{29}$ for Mn-doped $\alpha$-GeTe. However, in this paper the gap starts to saturate at larger amount of Mn impurity $(\sim 10 \%)$ and shows no decrease under further Mn concentration rise (at least up to 15\%). The saturation in the gap size with a further increase in Mn-concentration was associated in this work with Mn-phase segregation and corresponding antiferromagnetic coupling between neighboring Mn atoms that reduces the average FM moment per Mn atom.

Clustering of $\mathrm{V}$ somewhere in the sample volume instead of Bi substitution reduces the $\mathrm{V}$ concentration influencing Rashba bands, which can also lead to the reduction of the KP gap. Using STM and AFM we studied our V-doped BiTeI in order to validate the absence of impurity clusters. Fig. 1S in Suppl. Mat. shows the uniformity of the distribution of magnetic impurities and clearly demonstrates the absence of V-clusters for all studied V-concentrations.

\section{Theoretical analysis}

To further investigate the physics of the observed phenomena, we have theoretically analyzed the changes in the gap at the Kramers point with respect to the concentration of the magnetic metal dopant and on the temperature.

Let us consider the interface of BiTeI doped by magnetic ions as the system with two dimensional (2D) electron gas with spin-orbit (Rashba) interaction and the local dopant magnetic moments. Under the assumption that ions do not interact directly with each other but only with conduction electrons the Hamiltonian can be written as:

$$
\hat{H}=\hat{H}_{e}+J \sum_{i=1}^{N} \hat{S}_{i} \cdot \hat{s}_{i}
$$

where $\hat{H}_{e}$ - Hamiltonian of the electron system, $\hat{S}_{i}$ and $\hat{s}_{i}$ - spins of ions and electrons respectively, $N$ - the number of ions and $J$ - the exchange constant, which describes s-d interaction between free 2D Rashba electron gas and magnetic impurities system. Neglecting the fluctuations one can rewrite this expression in the following form:

$$
\hat{H}=\hat{H}_{e}+J\left\langle\hat{S}_{i}\right\rangle \sum_{i=1}^{N} \hat{s}_{i}+J\left\langle\hat{s}_{i}\right\rangle \sum_{i=1}^{N} \hat{S}_{i}-J N\left\langle\hat{S}_{i}\right\rangle\left\langle\hat{s}_{i}\right\rangle .
$$

In the mean field approximation one can obtain the decoupled Hamiltonian $H^{M F}=H_{e}^{M F}+H_{i}^{M F}-J N m_{e}^{\prime} M^{\prime}$, where $m_{e}^{\prime}=$ $m_{e} / g \mu_{B}, M^{\prime}=M / g_{i} \mu_{B}, m_{e}$ and $M$ - magnetizations of electron and ion subsystems respectively, $\mu_{B}$ - Bohr magneton, $g$ and

$g_{i}$ - g-factor of electron and ion respectively. The Hamiltonian of the ion subsystem has form $H_{i}^{M F}=J m_{e}^{\prime} \sum_{i=1}^{N} \hat{S}_{i}$, and the Hamiltonian of the electrons subsystem takes form:

$$
H_{e}^{M F}=\sum_{k} \psi_{k}^{+}\left(\hat{H}_{e}(k)+J M^{\prime} \frac{\sigma_{z}}{2}-\mu\right) \psi_{k},
$$

where $\sigma_{i}$ - Pauli matrices, $\mu$ - chemical potential and $\hat{H}_{e}(k)$ - one-electron Hamiltonian for BiTeI, which has the form:

$$
\hat{H}_{e}(k)=\frac{\hbar^{2} k^{2}}{2 m} \hat{I}+\alpha(\vec{k} \times \vec{z}) \vec{\sigma}
$$

where $m$ - effective mass of the electron, $\hbar$ - Dirac constant and $\alpha$ - the Rashba constant. Based on it, the electron energy can be written as:

$$
E_{e}^{ \pm}(k)=\frac{\hbar^{2} k^{2}}{2 m} \pm \sqrt{\Delta^{2}+\alpha^{2} k^{2}}-\mu
$$

where $\Delta=J M^{\prime} / 2$. The energy gap observed in the experiment in this case corresponds to $2 \Delta$.

Now let us consider two dimensional electron gas with this dispersion law. The mean energy (kinetic part) in this case can be written in form:

$$
F(\Delta)=\sum_{\vec{k} \sigma} E_{e}^{\sigma}(\vec{k}) \hat{n}_{k}=\sum_{\sigma} \int_{|\vec{k}| \leq k_{F}} E_{e}^{\sigma}(\vec{k}) f\left(E-E_{F}\right) \frac{k d k}{2 \pi} .
$$



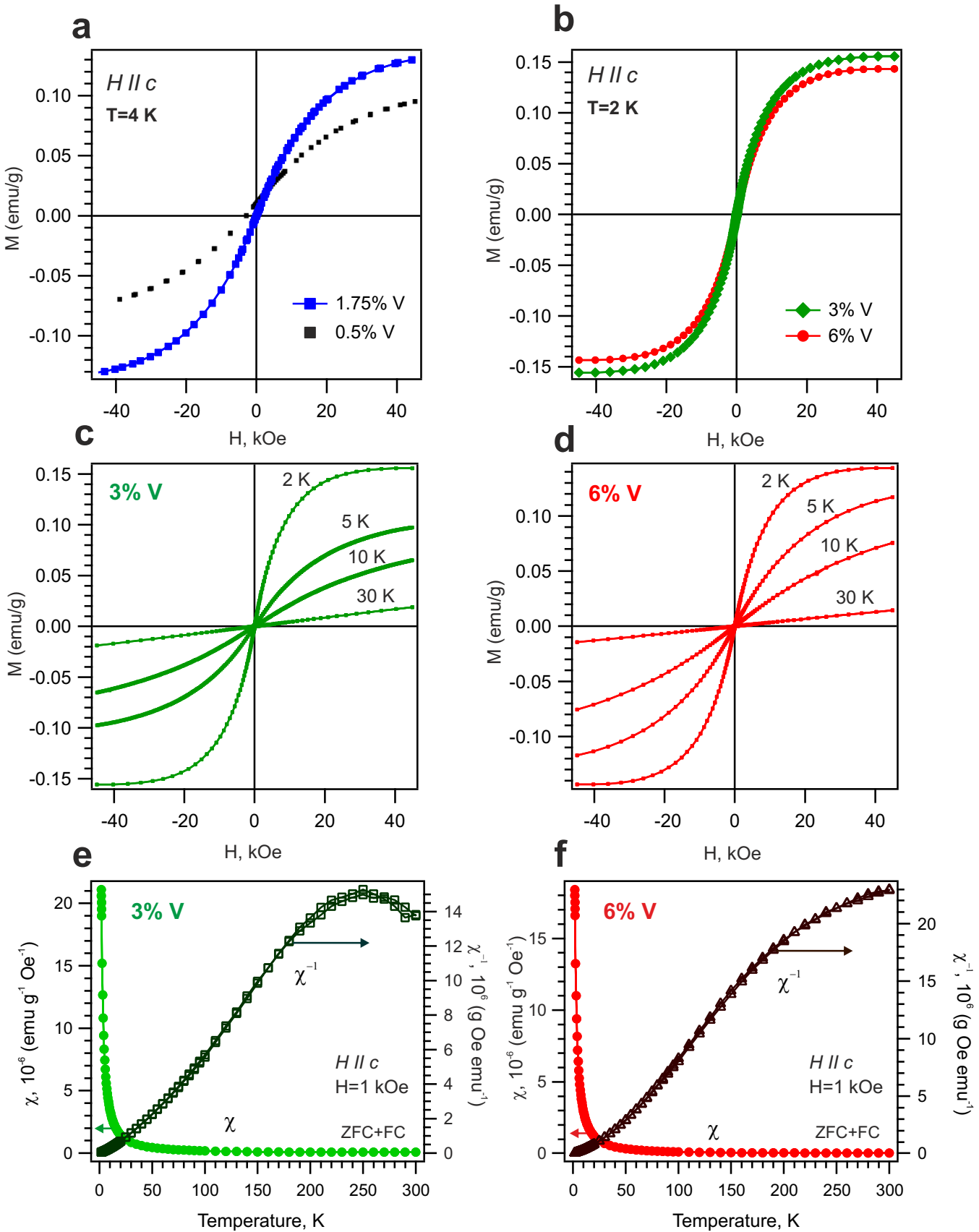

Figure 3. Comparative magnetization measurements using SQUID magnetometry. (a) - Magnetization curves $M(H)$ measured for $0.5 \% \mathrm{~V}$ and $1.75 \% \mathrm{~V}$-doped BiTeI at $4 \mathrm{~K}$ and (b) for $3 \% \mathrm{~V}$ and $6 \% \mathrm{~V}$-doped BiTeI at $2 \mathrm{~K}$. The external magnetic field was applied perpendicular to the surface plane. (c), (d) - Magnetization curves $M(H)$ at several temperatures for $3 \% \mathrm{~V}$ and $6 \% \mathrm{~V}$ -doped BiTeI, respectively. (e), (f) - Zero-field cooled (ZFC) and field cooled (FC) magnetic susceptibility curves $(\chi)$ and inverse magnetic susceptibility $\left(\chi^{-1}\right)$ as a function of temperature for V-concentrations of $3 \%$ and $6 \%$ with $1 \mathrm{kOe}$ field applied perpendicular to the surface plane. 
Similarly, the number of electrons can be written as $n_{e}=\sum_{\sigma} \int f\left(E-E_{F}\right) \frac{k d k}{2 \pi}$. Then neglecting corrections with order $T^{2}$ (low temperature approximation) one can calculate the mean energy as:

$$
\begin{aligned}
& F(\Delta)=\frac{\hbar^{2}}{16 \pi m}\left(k_{+}^{4}+k_{-}^{4}\right)+\frac{1}{6 \pi \alpha^{2}}\left(\left(\Delta^{2}+\alpha^{2} k_{+}^{2}\right)^{3 / 2}-\right. \\
& \left.-\left(\Delta^{2}+\alpha^{2} k_{-}^{2}\right)^{3 / 2}\right)-\frac{E_{F}}{4 \pi}\left(k_{+}^{2}+k_{-}^{2}\right),
\end{aligned}
$$

where $E_{F}$ - Fermi level, $k_{ \pm}$- the values of the wave vector given by the condition $E_{e}^{ \pm}\left(k_{ \pm}\right)=0$. Using the expression for the numbers of electrons one can write $4 \pi n_{e}=k_{+}^{2}+k_{-}^{2}$. Since experiment shows that the Fermi level is much higher than the gap (at the Kramers point), for the definition of $k_{ \pm}$we use the parabolic dispersion law $E_{e}^{ \pm}\left(k_{ \pm}\right) \approx \frac{\hbar^{2} k_{ \pm}^{2}}{2 m} \pm \alpha k_{ \pm}-E_{F}$. After simplifications, one can get:

$$
F(\Delta)=\text { const }-\frac{m}{2 \pi \hbar^{2}} \Delta^{2} .
$$

Then taking into account that $\Delta=\mu_{B} H$ one can write:

$$
m_{e}=-\frac{\partial F(H)}{\partial H}=\chi H=\chi \frac{J}{2 g_{i} \mu_{B}^{2}} M,
$$

where $\chi=m \mu_{B}^{2} / \pi \hbar^{2}$. It is also worth noting that in our case we have an analogue of the Pauli paramagnetism of free electrons, which appears not in the external, but in the exchange field. Indeed, the susceptibility in our case can be rewritten as $\chi=\frac{n_{e} \mu_{B}^{2}}{\left(E_{F}+m \alpha^{2} / \hbar^{2}\right)}$, while the Pauli susceptibility for $3 \mathrm{D}$ gas of free electrons has the form $\chi_{P}^{3 D}=\frac{3 n_{e} \mu_{B}^{2}}{2 E_{F}}$.

Now, knowing the magnetization of the electron system, we can consider the magnetization of the ionic system. We will assume that the ions do not interact with each other directly and are in the effective exchange field created by electron subsystem (which clearly follows from the form $H_{i}^{M F}$ ). Then their magnetization can be described by Brillouin function as:

$$
M=g_{i} \mu_{B} S_{i} N B_{S_{i}}\left(\frac{g_{i} \mu_{B} S_{i} H_{\text {exch }}}{T}\right),
$$

where $H_{\text {exch }}=J \frac{m_{e}}{g g_{i} \mu_{B}^{2}}$. Using it and $m_{e}$ one can rewrite dopant subsystem magnetization:

$$
M=g_{i} \mu_{B} S_{i} N B_{S_{i}}\left(\frac{\chi J^{2} S_{i}}{2 g g_{i} \mu_{B}^{3} T} M\right) .
$$

Considering the small magnetization $M$ near the Curie temperature, we can expand the Brillouin function in a Taylor series. This gives the critical temperature in the form:

$$
T_{C}=\frac{1}{6} \frac{\chi J^{2}}{g \mu_{B}^{2}} N S_{i}\left(S_{i}+1\right)
$$

This relation will make it possible to find the value of the exchange constant $J$ using experimental estimations of the critical temperature value $T_{C}$. After that, we can write down the dependence of the magnetization of ions in the region near $T_{C}$ :

$$
M=\frac{12 g g_{i} \mu_{B}^{4} T}{J^{3}} \sqrt{\frac{5 g\left(T_{C}-T\right)}{N \chi^{3} S_{i}\left(S_{i}+1\right)\left(2 S_{i}^{2}+2 S_{i}+1\right)}} .
$$

To consider the studied system not only near Curie temperature but also in the case of low temperatures instead of expanding the Brillouin function we can use the inverse Brillouin function in the form of a power series ${ }^{36}$. Using it, we can write the dependence $\mathrm{T}(\mathrm{M})$ :

$$
\frac{1}{T}=\sum_{i=0}^{+\infty} b_{2 i+1} \frac{2 g \mu_{B}^{2}}{N \chi J^{2}}\left(\frac{M}{g_{i} \mu_{B} N}\right)^{2 i}
$$

where $b_{2 i+1}$ - coefficients of the inverse Brillouin function expansion with $S_{i}$ from paper ${ }^{36}$. Taking into account that $M=$ $2 \Delta g_{i} \mu_{B} / J$ one can rewrite the expression (16) as:

$$
\frac{1}{T}=\sum_{i=0}^{+\infty} b_{2 i+1} \frac{2 g \mu_{B}^{2}}{N \chi^{2}}\left(\frac{2 \Delta}{J N}\right)^{2 i}
$$




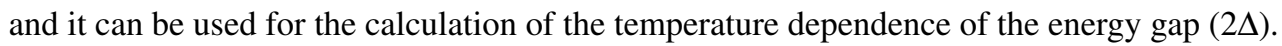

For the numerical estimations of $2 \Delta$ we consider $\mathrm{V}^{2+}$ impurities, which have $g_{i}=2$ and $S_{i}=3 / 2$ due to orbital quenching, and the impurity concentration $N=1.18 \times 10^{13} \mathrm{~cm}^{-2}$ which correspond to $2 \%$. We consider the first nine nonzero terms of the inverse Brillouin function expansion. The effective mass of the electron was estimated from ARPES experimental data as $m=1.43 \times 10^{-28} \mathrm{~g}$ (i.e. $\approx 0.157 \cdot m_{0}, m_{0}$ - electron mass). Using these parameters we varied the $T_{C}$ value to find the best coincidence between the theoretical energy gap dependence on the temperature and the experimental data. This procedure lead us to critical temperature estimation $T_{C} \approx 135 \mathrm{~K}$.
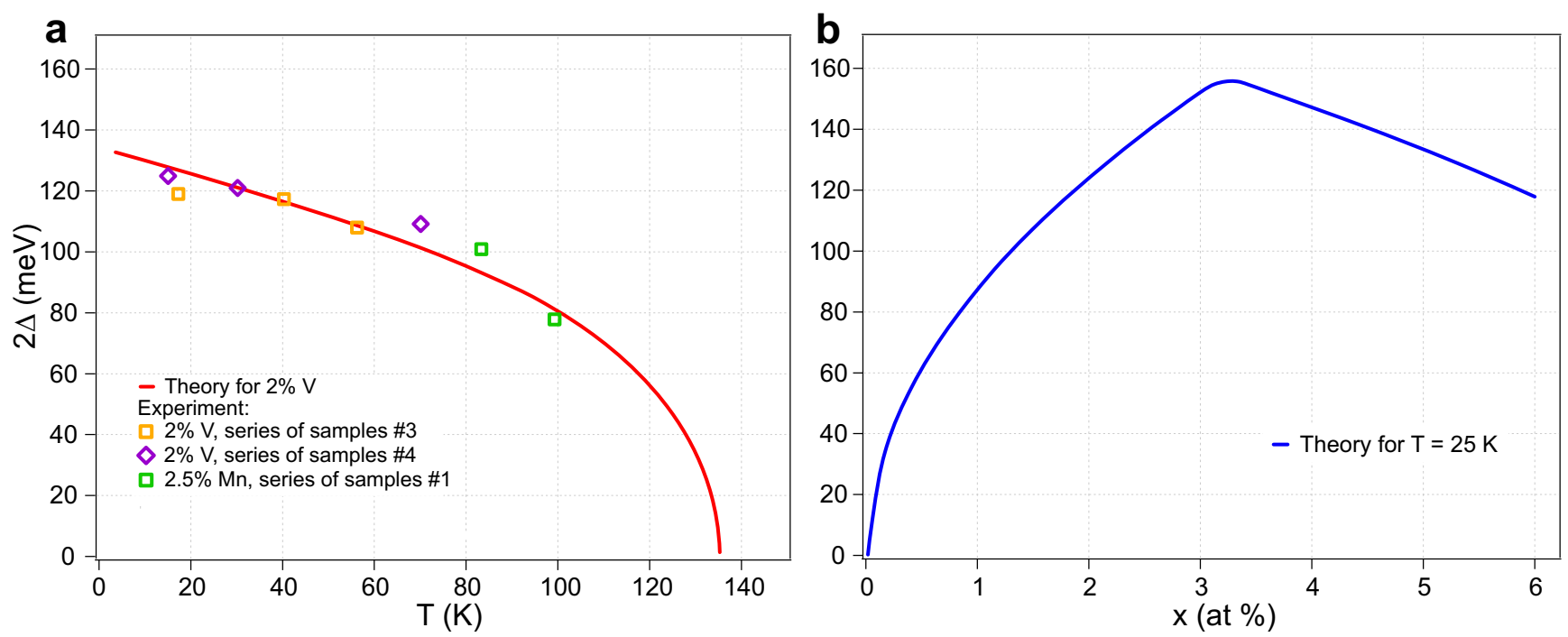

Figure 4. (a) - Theoretical dependence of the energy gap $2 \Delta$ on the temperature in comparison with experimental data for $2 \% \mathrm{~V}$ and $2.5 \% \mathrm{Mn}$ doping. (b) - Theoretical dependence of the energy gap on the concentration of magnetic impurities, taking into account possible pairing.

The theoretical dependence of the energy gap on temperature is shown in Fig. 4a together with the experimental points corresponding to the gap value obtained from the ARPES measurements, through the relative EDCs decomposition into spectral components presented in Figs. 2S-4S in Suppl. Mat., at various temperatures for 2\%V and 2.5\%Mn-doped BiTeI. One can see a slow decrease in the gap value with temperature for both types of magnetically-doped BiTeI with a tendency to close the gap at temperature above $130-140 \mathrm{~K}$. And at room temperature the gap is closed as it was shown in Ref. ${ }^{9}$. From Fig. 4 a the resulting theoretical dependence correlate very well with the experimental points.

Let us now consider the possibility of the formation of an antiferromagnetic pair by two impurities. Indeed, if two impurities are close enough then due to indirect exchange they will be oriented antiferromagnetically and therefore they will not contribute to the total magnetization.

We will assume that the size of the sample plate is $a \times b$, and the impurities interact if the distance between them is less than $r$. We will also assume that the first $N_{0}$ impurities do not interact with each other. The value $N_{0}$ can be estimated as $N_{0}=a b \kappa / \pi r^{2}$, where $\kappa$ is effective parameter, responsible for the uniform filling of the plate with impurities and lying in the range from 0 to 1 . Then, the probability of getting into the region inside a circle with radius $r$ of one of the $N_{0}$ impurities for the $\left(N_{0}+1\right)$-th impurity is equal to $p=w N_{0} \pi r^{2} / a b$, where $w$ is the degree of overlap of the circles with radius $r$ of the first $N_{0}$ impurities. If we introduce a random variable $x_{i}$, which takes on the value 0 when an impurity finds itself in the region with radius $r$ of other impurities and 1 - when it does not, the mathematical expectation of the sum of such random variables $S_{n}=\sum_{i=N_{0}}^{N} x_{i}$ will be equal to the number of new unpaired impurities in addition to $N_{0}$. But according to the central limit theorem, it is equal to the number of impurities in excess of $N_{0}$ (denote $N_{1}$ ) multiplied by the expectation of a single random variable $\mu\left(x_{i}\right)=\sum p_{i} x_{i}=1-w N_{0} \pi r^{2} / a b$. It should be noted that here to apply the central limit theorem we neglect the interaction between impurities in excess of $N_{0}$, i.e. we consider that $N_{0} \gg N_{1}$, and we consider that $N_{1} \gg 1$. Then the real number of unpaired impurities can be estimated as:

$$
N_{\text {real }}=N_{0}+N_{1}\left(1-\frac{w N_{0} \pi r^{2}}{a b}\right)-N_{1} \frac{w N_{0} \pi r^{2}}{a b} .
$$

The last term here represents the number of impurities from $N_{0}$, which no longer contribute to magnetization due to pairing with 
some of the $N_{1}$ impurities. Taking into account that the total number of impurities is $N=N_{0}+N_{1}$, one can get for $N>N_{0}$ :

$$
N_{\text {real }}=N(1-2 w \kappa)+2 w \kappa N_{0}
$$

Then, under the condition $w \kappa>0.5$, the real number of unpaired impurities begins to decrease after that the number of impurities exceeds $N_{0}$. In this case, $N_{\text {real }}$ should be substituted in all formulas for calculating $M$ and $2 \Delta$ instead of $N$. For numerical estimations let us consider $w \kappa=0.75, T=25 \mathrm{~K}$ so that the experimental estimation concentration related to $N_{0}$ is slightly about $3 \% \mathrm{~V}$ doping. The theoretical dependence of the energy gap on the number of impurities, taking into account possible pairing, is shown in Fig. $4 \mathrm{~b}$ and shows the observed non-monotonic variation. Therefore, from these results the assumption of a possible antiferromagnetic pairing between the impurities leads to a behaviour similar to that observed experimentally, thus supporting the proposed scenario.

\section{Conclusion}

We present experimental observation of non-monotonic dependence of the Kramers point band gap (KP gap) on magnetic dopant concentration in V-doped BiTeI using ARPES. The KP gap increases with V concentration up to $3 \%$ and reaches its maximum value of $130-135 \mathrm{meV}$ according to synchrotron-based ARPES. A further increase in V concentration leads to mitigation of the KP gap by $105-125 \mathrm{meV}$ depending on the sample. Estimations of the KP gap with the gapped Rashba and massive Dirac fermion models as well as measurements by means of laser-based ARPES also confirm the observed non-monotonic dependence. This can also be supported by the similar behaviour of the magnetization saturation value derived from SQUID measurements. However, the latter do not provide direct evidence of the magnetic origin of the KP as no sizable hysteresis loop in $M(H)$ curves is observed. Nevertheless, the results of ARPES and SQUID measurements can be rationalized under the condition of the presence of oppositely oriented magnetic domains at the nanoscale. They compensate each other in total, though locally they can lead to the KP gap opening. Theoretical analysis shows that the non-monotonic behavior can be explained by the increase of antiferromagnetic coupled atoms of magnetic impurities above a certain doping level. This leads to the reduction of the total magnetic moment at the domains and thus to the mitigation of the KP gap as observed in the experiment.

\section{Methods}

High-quality V- and Mn-doped BiTeI single crystals with various concentrations were synthesized using Bridgman method in Novosibirsk State University. The doping of V (Mn)-impurities is given in atomic percentages where V (Mn) magnetic atoms mainly replace the $\mathrm{Bi}$ atoms. The level $x \%$ of $\mathrm{V}(\mathrm{Mn})$ doping corresponds to stoichimetry of $\mathrm{Bi}_{1-0.0 x} \mathrm{~V}(\mathrm{Mn})_{0.0 x} \mathrm{TeI}$. Clean sample surfaces were obtained by ultrahigh vacuum cleavage.

The measurements of ARPES dispersion maps were carried out at the BaDElPh beamline at Elettra (Trieste, Italy), the BL-9 beamline at HiSOR (Hiroshima, Japan), the One-cube end station and RGBL-2 end station at BESSY II (Helmholtz-Zentrum Berlin, Germany), spin-ARPES end station at MAX-Lab (Lund, Sweden) using synchrotron radiation with photon energy in the regions 20-25 eV and at the $\mu$-Laser ARPES system $(h v=6.3 \mathrm{eV})$ at HiSOR (Hiroshima, Japan) with improved angle and energy resolution and a high space resolution of the laser beam (spot diameter around $5 \mu \mathrm{m}$ ). The spectra were measured using a Scienta R4000 or a Specs Phoibos 150 analyzer with an incidence angle of the laser and synchrotron radiation of $50^{\circ}$ relative to the surface normal at temperatures between 1 and $20 \mathrm{~K}$.

In Figs. 1.d,e. and Fig. 4 the series of ARPES measurements results denoted by colored points are presented. In Figs. 1.d,e the Series \#1 corresponds to the 0.5\% V-doped BiTeI samples, measured at BESSY II; the Series \#2 to 0.5\% V-doped BiTeI measured at MAX-Lab; the Series \#3 to 2\%V-doped BiTeI measured at Elettra; the Series \#4 to 2\%V-doped BiTeI measured at HiSOR; the Series \#5 to 3\%V and 6\%V-doped BiTeI measured at BESSY II; the Series \#6, \#7 to 6\%V-doped BiTeI measured at Elettra. In Fig. 4 the sample Series \#4 and \#3 corresponds to the 2\%V-doped BiTeI samples measured at HiSOR and Elettra, respectively. The sample Series \#1 in Fig. 4 was measured for 2.5\%Mn-doped BiTeI at MAX-Lab. The base pressure during all photoemission experiments was better than $1-2 \times 10^{-11}$ mbar.

The photoelectron spectra (EDCs) at $k_{\|}=0 \AA^{-1}$ obtained from the ARPES dispersion maps were decomposed into components by fitting procedure for the analysis of the gap size at the Kramers point. We optimized the fit by two main components at the Kramers point, the asymmetric component near the Fermi level corresponding to the features of iodine electronic states, and the small component at higher binding energy due to the features of the valence band states. The line shape and the width of the two main components were defined by the fitting procedure of the parabolic branches. Raw EDC data are shown in figure 1 by circles along with the best-fit results, the corresponding components and the background.

Measurements of magnetic properties were carried out in the Resource Center "Center for Diagnostics of Materials for Medicine, Pharmacology and Nanoelectronics" of SPbU Research Park using a SQUID magnetometer with a helium cryostat manufactured by Quantum Design. The experiments were performed in a pull mode in terms of temperature and magnetic 
field. The magnetic field was applied perpendicular to the sample surface. STM images were obtained using a Omicron VT SPM in the Resource Center "Physical Methods of Surface Investigation" of SPbU Research park at room temperature. AFM measurements were carried out under atmospheric conditions and at room temperature in the tapping mode on a NT-MDT Solver Pro-M microscope at the SPbU laboratory.

\section{References}

1. Sau, J. D., Lutchyn, R. M., Tewari, S. \& Sarma, S. D. Generic new platform for topological quantum computation using semiconductor heterostructures. Phys. review letters 104, 040502 (2010).

2. Baltz, V. et al. Antiferromagnetic spintronics. Rev. Mod. Phys. 90, 015005 (2018).

3. Ishizaka, K. et al. Giant Rashba-type spin splitting in bulk BiTeI. Nat. materials 10, 521-526 (2011).

4. Maaß, H. et al. Spin-texture inversion in the giant Rashba semiconductor BiTeI. Nat. Commun. 7, 1-7 (2016).

5. Eremeev, S., Nechaev, I. \& Chulkov, E. V. Giant Rashba-type spin splitting at polar surfaces of BiTeI. JETP letters 96, 437-444 (2012).

6. Eremeev, S. V., Nechaev, I. A., Koroteev, Y. M., Echenique, P. M. \& Chulkov, E. V. Ideal two-dimensional electron systems with a giant Rashba-type spin splitting in real materials: surfaces of bismuth tellurohalides. Phys. review letters $\mathbf{1 0 8}$, 246802 (2012).

7. Landolt, G. et al. Disentanglement of surface and bulk Rashba spin splittings in noncentrosymmetric BiTeI. Phys. review letters 109, 116403 (2012).

8. Crepaldi, A. et al. Giant ambipolar Rashba effect in the semiconductor BiTeI. Phys. review letters 109, 096803 (2012).

9. Klimovskikh, I. I. et al. Giant magnetic band gap in the Rashba-split surface state of Vanadium-doped BiTeI: A combined photoemission and ab initio study. Sci. reports 7, 1-8 (2017).

10. Chen, Y. et al. Massive Dirac fermion on the surface of a magnetically doped topological insulator. Science 329, 659-662 (2010).

11. Xu, S.-Y. et al. Hedgehog spin texture and Berry's phase tuning in a magnetic topological insulator. Nat. Phys. 8, 616-622 (2012).

12. Shikin, A. M. et al. Signatures of in-plane and out-of-plane magnetization generated by synchrotron radiation in magnetically doped and pristine topological insulators. Phys. Rev. B 97, 245407 (2018).

13. Shikin, A. et al. Dirac gap opening and Dirac-fermion-mediated magnetic coupling in antiferromagnetic Gd-doped topological insulators and their manipulation by synchrotron radiation. Sci. reports 9, 1-17 (2019).

14. Shikin, A. et al. Gap Opening Mechanism at the Dirac Point in the Electronic Spectrum of Gd-Doped Topological Insulator. Phys. Solid State 62, 338-349 (2020).

15. Shikin, A. M. et al. Nature of the Dirac gap modulation and surface magnetic interaction in axion antiferromagnetic topological insulator $\mathrm{MnBi}_{2} \mathrm{Te}_{4}$. Sci. Reports 10, 13226, DOI: 10.1038/s41598-020-70089-9 (2020).

16. Estyunin, D. A. et al. Signatures of temperature driven antiferromagnetic transition in the electronic structure of topological insulator $\mathrm{MnBi}_{2} \mathrm{Te}_{4}$. APL Mater. 8, 021105, DOI: 10.1063/1.5142846 (2020).

17. Ogawa, N., Bahramy, M., Murakawa, H., Kaneko, Y. \& Tokura, Y. Magnetophotocurrent in BiTeI with Rashba spin-split bands. Phys. Rev. B 88, 035130 (2013).

18. Kovács-Krausz, Z. et al. Electrically controlled spin injection from giant Rashba spin-orbit conductor BiTeBr. Nano Lett. (2020).

19. Ogawa, N., Bahramy, M., Kaneko, Y. \& Tokura, Y. Photocontrol of Dirac electrons in a bulk Rashba semiconductor. Phys. Rev. B 90, 125122 (2014).

20. Koga, T., Nitta, J., Takayanagi, H. \& Datta, S. Spin-Filter Device Based on the Rashba Effect Using a Nonmagnetic Resonant Tunneling Diode. Phys. Rev. Lett. 88, 126601 (2002).

21. Sinova, J. et al. Universal Intrinsic Spin Hall Effect. Phys. Rev. Lett. 92, 126603 (2004).

22. Kovács-Krausz, Z. et al. Electrically Controlled Spin Injection from Giant Rashba Spin-Orbit Conductor BiTeBr. Nano Lett. 20, 4782-4791 (2020).

23. Kitaev, A. Y. Unpaired Majorana fermions in quantum wires. Physics-Uspekhi 44, 131 (2001). 
24. Tewari, S., Stanescu, T. D., Sau, J. D. \& Sarma, S. D. Topologically non-trivial superconductivity in spin-orbit-coupled systems: bulk phases and quantum phase transitions. New J. Phys. 13, 065004 (2011).

25. Fu, L. \& Kane, C. L. Superconducting proximity effect and Majorana fermions at the surface of a topological insulator. Phys. review letters 100, 096407 (2008).

26. Alicea, J., Oreg, Y., Refael, G., von Oppen, F. \& Fisher, M. P. A. Non-Abelian statistics and topological quantum information processing in 1D wire networks. Nat. Phys. 7, 412-417 (2011).

27. Nadj-Perge, S., Drozdov, I. K., Bernevig, B. A. \& Yazdani, A. Proposal for realizing Majorana fermions in chains of magnetic atoms on a superconductor. Phys. Rev. B 88, 020407 (2013).

28. Shikin, A. et al. Anomalously large gap and induced out-of-plane spin polarization in magnetically doped 2D Rashba system: V-doped BiTeI. 2D Mater. 4, 025055 (2017).

29. Krempaskỳ, J. et al. Entanglement and manipulation of the magnetic and spin-orbit order in multiferroic Rashba semiconductors. Nat. communications 7, 1-7 (2016).

30. Krempaskỳ, J. et al. Spin-resolved electronic structure of ferroelectric $\alpha$-GeTe and multiferroic Ge1- xMnxTe. J. Phys. Chem. Solids 128, 237-244 (2019).

31. Zhang, P. et al. A precise method for visualizing dispersive features in image plots. Rev. Sci. Instruments 82, 043712, DOI: 10.1063/1.3585113 (2011).

32. Iwasawa, H. et al. Development of laser-based scanning $\mu$-ARPES system with ultimate energy and momentum resolutions. Ultramicroscopy 182, 85-91 (2017).

33. Bean, C. \& Livingston, u. D. Superparamagnetism. J. Appl. Phys. 30, S120-S129 (1959).

34. Bean, C. Hysteresis loops of mixtures of ferromagnetic micropowders. J. Appl. Phys. 26, 1381-1383 (1955).

35. Ishikawa, Y. Superparamagnetism in magnetically dilute systems. J. Appl. Phys. 35, 1054-1059 (1964).

36. Katriel, J. Continued-Fraction Approximation for the Inverse Brillouin Function. physica status solidi (b) 139, 307-310 (1987).

\section{Acknowledgements}

The authors acknowledge support by the Ministry of Science and Higher Education of the Russian Federation (Grant No 075-15-2020-797 (13.1902.21.0024)). The authors acknowledge the HiSOR, BESSY II, Elettra and SPbU Research Park staff for technical supports during the experiments and supplying the liquid Helium.

\section{Author contributions statement}

The manuscript was written by A.M.Sh., A.A.R., D.A.E. and P.N.S. All co-authors took part in the discussion and analysis of the experimental results. Preparation of the manuscript for publication with the presentation of the figures was carried out by A.A.R and D.A.E. ARPES measurements were carried out by I.I.K., D.A.E., A.M.Sh., S.O.F., A.A.R., A.G.R., V.Yu.V., L.P., G.D.S., S.K., O.E.T., and A.K. Measurements of magnetic properties were carried out by E.V.Sh, A.V.K., A.A.R., I.I.K. The STM and AFM analysis was done by A.G.R., A.E.P. and S.O.F. Theoretical analysis was performed by P.N.S., A.K.Z and K.A.Z. Crystals were grown with following testing their crystalline structure by K.A.K and O.E.T. The project was planned by A.M.Sh., I.I.K. and A.A.R. All authors reviewed the manuscript.

\section{Competing interests}

The authors declare that they have no competing interests.

\section{Additional information}

The authors declare that the data supporting the findings of this study are available within the paper. 


\section{Supplementary Files}

This is a list of supplementary files associated with this preprint. Click to download.

- Supplementarymaterial08082021.pdf 